\title{
Evaluation of Methods to Characterize the Change of the Respiratory Sinus Arrhythmia with Age in Sleep Apnea Patients
}

\author{
John F Morales ${ }^{1,2}$, Margot Deviaene ${ }^{1,2}$, Javier Milagro ${ }^{3}$, Dries Testelmans ${ }^{4}$, Bertien Buyse ${ }^{4}$, Rik Willems ${ }^{5}$, \\ Michele Orini $^{6,7}$, Sabine Van Huffel ${ }^{1,2}$, Raquel Bailón ${ }^{3}$, Carolina Varon ${ }^{1,2}$
}

\begin{abstract}
The High Frequency (HF) band of the power spectrum of the Heart Rate Variability (HRV) is widely accepted to contain information related to the respiration. However, it is known that this often results in misleading estimations of the strength of the Respiratory Sinus Arrhythmia (RSA). In this paper, different approaches to characterize the change of the RSA with age, combining HRV and respiratory signals, are studied. These approaches are the bandwidths in the power spectral density estimations, bivariate phase rectified signal averaging, information dynamics, a time-frequency representation, and a heart rate decomposition based on subspace projections. They were applied to a dataset of sleep apnea patients, specifically to periods without apneas and during NREM sleep. Each estimate reflected a different relationship between RSA and age, suggesting that they all capture the cardiorespiratory information in a different way. The comparison of the estimates indicates that the approaches based on the extraction of respiratory information from HRV provide a better characterization of the age-dependent degradation of the RSA.
\end{abstract}

\section{INTRODUCTION}

The Respiratory Sinus Arrhythmia (RSA), initially described in 1733 [1], can be seen as an increased Heart Rate (HR) during inspiration and a decreased HR during expiration. Since its discovery, its function and physiological mechanisms have remained unclear. The most accepted hypothesis suggests that the RSA helps to match the perfusion and ventilation processes during the respiratory cycle in order to improve the pulmonary gas exchange and optimize the energy efficiency of the pulmonary circulation. Nevertheless, some studies suggest that more tests are needed to prove this hypothesis [2]. The controversy around the role of the RSA highlights the importance of methods for its quantification. To observe the modulation exerted by the respiration on the HR, a Heart Rate Variability (HRV) representation can be used. The respiratory modulation is generally reflected within

\footnotetext{
${ }^{1,2}$ John F Morales, Margot Deviaene, Sabine Van Huffel and Carolina Varon are with ESAT - STADIUS, Stadius Centre for Dynamical Systems, Signal Processing and Data Analytics and with IMEC, KU Leuven, 3000 Leuven, Belgium, (jmorales; margot.deviaene; sabine.vanhuffel; carolina.varon(esat.kuleuven.be)

${ }^{3}$ Javier Milagro and Raquel Bailón are with the Biomedical Signal Interpretation and Computational Simulation (BSICoS) group, University of Zaragoza, 50018 Zaragoza, Spain, (milagro; rbailon@unizar.es)

${ }^{4}$ Dries Testelmans and Bertien Buyse are with the Department of Pneumology, UZ Leuven, 3000 Leuven, Belgium, (dries.testelmans; bertien. buyse@uzleuven.be)

${ }^{5}$ Rik Willems is with the Department of Cardiology, UZ Leuven, 3000 Leuven, Belgium, (rik.willems@uzleuven.be)

${ }^{6,7}$ Michele Orini is with the Department of Mechanical Engineering, University College London, London WC1E 6BT, U.K., and also with Barts Heart Centre, St Bartholomews Hospital, University College London, London WC1E 6BT, U.K., (m.orini@ucl.ac.uk)
}

the High Frequency (HF) band $(0.15 \mathrm{~Hz}-0.4 \mathrm{~Hz})$ of the Power Spectral Density (PSD) estimate of the HRV. However, it has also been observed that the influence of the respiratory rate might fall within bands outside the HF band or it might consist of a narrow spectrum inside the HF band. Hence, quantifying the RSA as the total power contained in the HF of the HRV might be misleading [3].

The aim of this work was, on the one hand, to compare different state-of-the-art methods typically used to quantify the RSA, and on the other hand, to assess whether they all capture the same type of information. This evaluation was done under the assumption that the strength of the RSA decreases with age [4]. The features were assessed in terms of their capability to characterize this change. The methods compared here analyze the information shared between the respiratory signals and the HRV representation (i.e. the tachogram). The breathing signals were used either to separate the respiratory information from the HRV, to define frequency bands different from the HF band, or to quantify the information transferred from respiration to HRV. To this end, the following approaches were used:

- The Bandwidth $(B W)$ of the respiration was used to define the frequency bands of interest in the PSD of the $\mathrm{HRV}$, as produced by the respiratory modulation. The normalized power in this band was evaluated as RSA quantification [3].

- Entropy measures were used to quantify the information transfer from respiration to HRV [5].

- Bivariate Phase Rectified Signal Averaging (BPRSA) curves were calculated and used to derive features as possible RSA quantifications [6].

- A Time-Frequency representation was used to separate the spectrum of the HRV in two components. One of them corresponding to the respiratory modulation. The total power contained in this component was included in the evaluation [7].

- Subspace projections were used to extract the respiratory information from the HRV. This information was then used to quantify the total power of the HRV explained by the respiration [8].

These estimates were compared with the normalized power in the HF band $\left(H F_{n}\right)$ of the HRV [9].

\section{MATERIALS AND METHODS}

\section{A. Materials}

A dataset with electrocardiogram (ECG) and thoracic Respiratory Inductive Plethysmography (RIP) signals was 
used. It contains Polysomnography (PSG) recordings of 110 patients with Obstructive Sleep Apnea (OSA) and different OSA-associated comorbidities. The signals were acquired with a sampling frequency of $500 \mathrm{~Hz}$. The apneas and sleep stages were annotated by sleep specialists according to the AASM 2012 scoring rules [10]. The OSA severity was assessed with the Apnea Hypopnea Index (AHI), i.e. average number of respiratory events per hour of sleep. 100 of the patients were first divided in two groups: one containing 50 OSA patients (AHI>15) without comorbidities and another with 50 OSA patients with comorbidities (hyperlipidemia: 49, hypertension: 40, diabetes: 5, myocardial infarction: 4 and stroke: 2). These groups were matched by age, gender and Body Mass Index (BMI). 33 of the 50 patients with comorbidities were taking medication to control the blood pressure at the moment of the recordings. The remaining 10 subjects $(\mathrm{AHI}<15)$ did not present comorbidities. 27 of the patients in the dataset had smoked or were smokers at the moment of the recordings. The demographics are summarized in Table I.

\section{B. Preprocessing and segment extraction}

The R-peaks in the ECG signals were detected using the algorithm proposed in [11]. Next, the R-peaks were visually corrected for mis-detections and ectopic beats. Subsequently, an HRV representation $\left(d_{R R}\right)$ with a sampling frequency of $4 \mathrm{~Hz}$ was calculated using the RR-interval series and a cubic spline interpolation. Furthermore, the respiratory signals were downsampled to $4 \mathrm{~Hz}$. Finally, both $d_{R R}$ and RIP signals were bandpass filtered $(0.05 \mathrm{~Hz}-1 \mathrm{~Hz})$ with a zero phase distortion to remove baseline and high frequency artifacts.

After preprocessing, segments were extracted from each subject. To this end, the duration of the annotated apneas was first extended to one minute. This was done to eliminate possible biases generated by the recovery period after an apneic event [12]. Afterwards, epochs of 5 minutes were extracted. Next, only those during non-Rapid Eye Movement (NREM) sleep and without apneas were selected. Finally, epochs containing either a severely contaminated ECG signal or an irregular respiratory signal were removed after visual inspection. As a result, different number of segments were available for each patient.

\section{RSA quantification}

Features to quantify the RSA were calculated on the 5minutes apnea-free epochs. Afterwards, the estimates of segments from the same patient were averaged. This produced an estimation of the strength of the RSA per subject. The features were derived using the following approaches:

1) Respiratory Bandwidth $(B W)$ : The PSDs of the respiratory and $d_{R R}$ signals were computed using the Welch's algorithm with a hamming window of $40 s$ with $20 s$ overlap. Afterwards, the RSA was quantified by first finding the frequency band corresponding to the $-3 d B$ bandwidth in the respiratory signal. Next, the influence of the respiration on $d_{R R}$ was quantified as the normalized power contained in
TABLE I

Demographics. THE AGE, BMI AND AHI ARE GIVEN AS THE MEAN VALUES \pm THE STANDARD DEVIATION. BELOW ARE THE RANGES GIVEN AS: $\left(25^{\text {th }}\right.$ PERCENTILE $-75^{\text {th }}$ PERCENTILE, MINIMA - MAXIMA)

\begin{tabular}{cccccc}
\hline \hline \multirow{2}{*}{$\mathrm{N}$} & $\begin{array}{c}\text { Age } \\
\text { Years }\end{array}$ & $\begin{array}{c}\text { BMI } \\
\mathrm{Kg} / \mathrm{m}^{2}\end{array}$ & $\begin{array}{c}\text { AHI } \\
\text { Events/h }\end{array}$ & Gender & Smoking \\
\hline \multirow{2}{*}{110} & $47.3 \pm 10.6$ & $29.3 \pm 4.6$ & $37.8 \pm 23.8$ & M: 82 & Y: 26 \\
& $(38-55,26-68)$ & $(25.9-32.8,20.7-44.7)$ & $(21.4-53.25,1.8-111.4)$ & W: 28 & N: 83 \\
\hline \hline
\end{tabular}

the PSD of $d_{R R}$ in the same frequency band, and denoted by $P_{B W}$ [3]. This method was also used to calculate the respiratory rate as given by the peak frequency in the PSD of the respiration.

2) Information Dynamics: The methods developed under the framework of information dynamics can be used to estimate the information stored in the respiratory and the $d_{R R}$ signals, and the information transfer between them. One possible information estimation is the entropy. Different entropy terms exist in the literature [13]. The Cross Entropy $\left(C_{E}\right)$, i.e. the information shared between $d_{R R}$ and the past information of the respiration [5], was chosen since this term is related with the influence of the respiration on $d_{R R}$.

3) Bivariate Phase Rectified Signal Averaging (BPRSA): BPRSA curves were used to identify quasi periodicities in $d_{R R}$ (target signal) caused by changes in the respiratory signal (driver signal). The procedure to derive these curves consists of first locating anchor points, that correspond to either increasing or decreasing points, in the driver signal [6]. Afterwards, portions of the target signal are defined in windows around the anchor points. Finally, these portions are averaged to obtain the PRSA curve. This curve reflects the quasi periodicities occurring in the target signal due to changes in the driver signal. In this paper, 2 features were derived from the PRSA curves [14], namely the RMS value when the anchor points are the increasing points $\left(R M S^{+}\right)$and the RMS values when the anchor points are the decreasing points $\left(R M S^{-}\right)$.

4) Time Frequency (TF) Representation: Spectrograms based on a Cohens class TF distribution as described in [7] were built for each 5-min segment. This tool allows to visualize changes in the frequency content of the signals over time while reducing biases in the estimations that occur with other TF representations. In addition, the coherence spectrum between the signals, which represents the shared frequency content and how this changes over time, was calculated using the same TF representation. In [7], it was shown that the product between the coherence spectrum and the spectrogram of $d_{R R}$ can be used to extract the spectrum of the respiratory component. After this extraction, the respiratory spectrum was normalized by the spectrum of $d_{R R}$ and then averaged to obtain a quantification of the RSA $\left(P_{T F}\right)$ per segment.

5) Subspace projections: A subspace projection is a method that can be used to separate the respiratory information from $d_{R R}$ by projecting the latter onto a respiratory subspace [8]. This projection produced a time series $d_{\text {Resp }}$ describing all dynamics of $d_{R R}$ linearly explained by the respiration. These time series were then used to calculate 
the relative power of the respiratory influences as $P_{s u b}=$ $\left(d_{\text {Resp }}^{T} d_{\text {Resp }}\right) /\left(d_{R R}^{T} d_{R R}\right)$.

\section{Comparison}

The RSA estimates were compared using linear regression between each estimate and the age of the subjects. Each regression was evaluated based on the $R_{a d j}^{2}$, the correlation coefficients (Pearson $\left(\rho_{p}\right)$ and Spearman $\left(\rho_{s}\right)$ ) and their significance $(p<0.05)$. The features with higher correlation coefficients were hypothesized to better characterize the expected change of RSA with age.

In addition, the patients were grouped in bins of 10 years and significant differences between the groups were tested using the Kruskal-Wallis test $(p<0.05)$ with Bonferroni correction for multiple comparisons.

Furthermore, multiple linear regression models between age and all possible combinations of the variables AHI, BMI, presence of cardiac comorbidities, medication intake, gender and smoking (yes or no) were made in order to analyze possible confounding effects.

Finally, it has been observed that the strength of the RSA might depend on the respiratory rate [15]. For this reason, regression and correlation coefficients between the respiratory rate with the age were computed to evaluate this variable as a confounder.

\section{RESULTS AND DISCUSSION}

The different regressions between age and the possible confounding variables did not produce a $R_{a d j}^{2}$ higher than 0.06 when a single variable was included in the regression. Furthermore, the combined effects of variables did not produce a $R_{a d j}^{2}$ higher than 0.17. Hence, the AHI, BMI, presence of cardiac comorbidities, medication intake, gender and smoking are not linearly related to the age of the subjects in this dataset. In addition, $\rho_{s}$ was computed for age with the AHI and BMI variables to check for possible non-linear confounding effects. Only the $\rho_{s}$ for the BMI was significant but weak $\left(\rho_{s}=-0.21, p=0.02\right)$. These results suggest that the effect of these confounding variables is negligible.

After preprocessing the signals and extracting the 5-minute epochs, 8 patients were removed from the analysis since less than 5 segments were available for them. The RSA estimates and the respiratory rates were calculated for the remaining patients.

Afterwards, the effect of the respiratory rate on the RSA amplitude as a confounder was evaluated. There are studies supporting [15] and contradicting [16] this hypothesis. In this study, the regression coefficients and correlations for the average respiratory rate per patient with age were not significant. This result suggests that the degradation of the RSA with age in this dataset does not depend on the respiratory frequency. However, it was also observed that each subject breathes within a narrow range of frequencies during the night. It is possible that the strength of the RSA would change within each subject if they breathe with a
TABLE II

$R_{a d j}^{2}, \rho_{p}$ AND $\rho_{s}$ FOR THE DIFFERENT RSA ESTIMATES.

\begin{tabular}{l|c|ccc}
\hline \hline Estimation & Method & $R_{a d j}^{2}$ & $\rho_{p}$ & $\rho_{s}$ \\
\hline$P_{T F}$ & TF-Rep & 0.372 & 0.615 & 0.602 \\
$P_{B W}$ & PSD & 0.315 & 0.568 & 0.566 \\
$P_{s u b}$ & Subspace & 0.313 & 0.566 & 0.552 \\
$C_{E}$ & Entropy & 0.310 & 0.564 & 0.544 \\
$R M S^{+}$ & BPRSA & 0.271 & 0.528 & 0.539 \\
$R M S^{-}$ & BPRSA & 0.199 & 0.455 & 0.468 \\
$H F_{n}$ & Standard & 0.184 & 0.439 & 0.435 \\
\hline \hline
\end{tabular}

wider range of respiratory rates. Hence, the results suggest that the respiratory rate is not a confounder in this specific case, but conclusions can not be made on the relationship between RSA and respiratory frequency.

Figure 1 shows the boxplots per age group for some of the features and the significant differences between them. The boxplots for the $P_{s u b}$ and $R M S^{+}$features are not included since the significant differences were similar to those for $P_{T F}$ and $R M S^{-}$, respectively. Table II summarizes the results for the regression coefficients. These were significant $(p \ll 0.05)$ in all cases. The $\rho_{p}$ and $\rho_{s}$ coefficients did not differ significantly, suggesting that the relationship between the estimators and the age of the patients is linear.

The $P_{T F}$ is the feature that better captures the changes produced by age $\left(\rho_{p}=0.615, p \ll 0.05\right)$ according to table II. In addition, Figure 1 shows that this feature is the one able to produce the most significant differences between the age groups. This feature, and the $P_{s u b}$ are based on the extraction of the respiratory modulation from $d_{R R}$ and the quantification of the fraction of power that it contains. These results suggest that the degradation of the respiratory modulation in the elderly is reflected in the whole spectrum of $d_{R R}$. These estimators might describe better the change of RSA with Age than the $H F_{n}$ since they only consider information contained in $d_{R R}$ produced by the respiration. Instead, the $H F_{n}$ includes information due to other processes that might be redundant in terms of the quantification of the RSA.

The $P_{B W}$ feature is the second best one to characterize the degradation of the RSA with age according to the correlation coefficients. It reflects more significant differences between the age groups compared to the $H F_{n}$. This might be due to the occurrence of the respiratory rate in bands narrower than the HF and this is better captured when the spectrum of the respiration is considered in the calculations, as explained in [3].

On the other hand, $C_{E}$ captures information related to the predictability of the heart rate taking into account its own past and the past of the respiratory signal. From the correlation and the significant differences between age groups in Figure 1, it is observed that this predictability is reduced in the elderly population, suggesting that the role of other modulators of heart rate, different from respiration, becomes stronger with age.

Finally, the RMS values derived from the BPRSA curves can be interpreted as a measure of the effect that changes in 

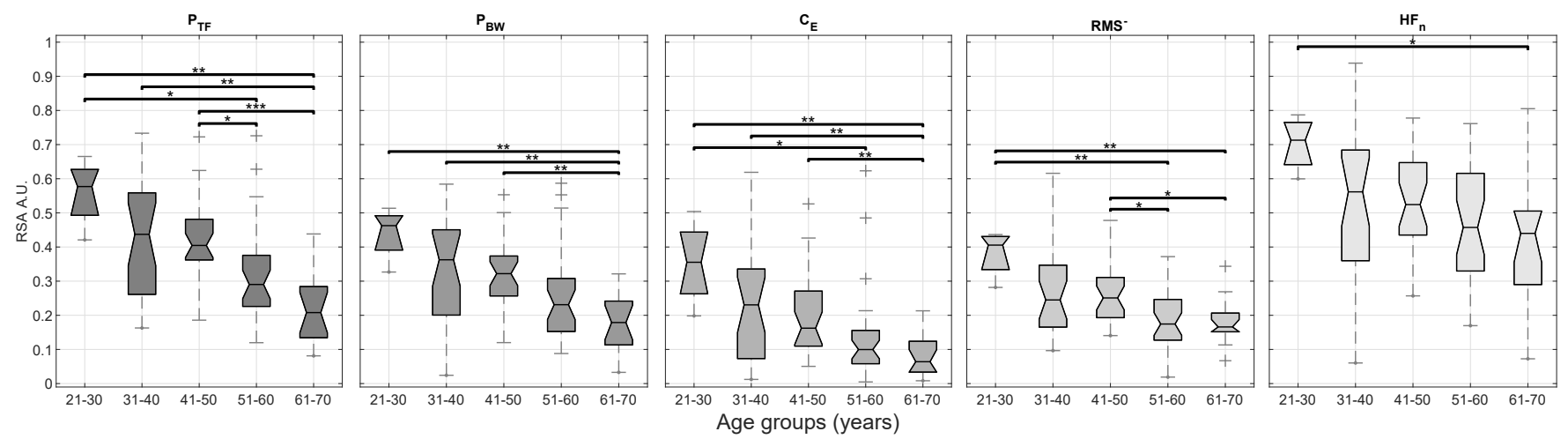

Fig. 1. Boxplots for different age groups. The number of patients per group were, 4 (21-30), 26 (31-40), 27 (31-40), 29 (31-40) and 16 (31-40).

respiration have on the dynamics of the heart rate. The fact that the $R M S^{+}$feature has a higher correlation coefficient with age than the $R M S^{-}$suggests that the efficiency of the cardiorespiratory interactions is more affected during inhalation than during exhalation in the elderly. However, the metrics for both features are very similar to conclude this. In addition, these features found the same significant differences between the age groups, suggesting that they characterize the degradation of the RSA in the same way. It is important to highlight that the selected segments only contain clean ECG and respiratory signals with a regular pattern during NREM sleep and without any apnea episodes. Hence, the results might be different in cases in which irregular respiratory rates occur, e.g. in the presence of apneas. In these cases, the $B W$ of the respiration would be wider and probably more frequencies in the spectrum of $d_{R R}$ would interact with the respiration. In addition, the comparisons in this paper were done based on the correlation coefficients, but all of them reflect weak to moderate relationships between the estimators and the age of the patients.

\section{CONCLUSIONS}

In this study, different approaches to characterize agedependent changes in RSA were compared. The results indicate that the quantification of age-dependent changes in RSA using the normalized power in the HF band might not be accurate. Alternative methods are shown to be better and should be considered when analyzing the RSA. In particular, the power of the HRV explained by respiration and estimated with Time-Frequency methods was the best feature to explain the age-dependent changes of RSA.

\section{ACKNOWLEDGMENT}

Agentschap Innoveren \& Ondernemen (VLAIO): STW 150466 OSA+. European Research Council. The research leading to these results has received funding from the European Research Council under the European Union's Seventh Framework Programme (FP7/2007-2013) / ERC Advanced Grant: BIOTENSORS ( ${ }^{\circ}$ 339804)/ TARGID - Development of a novel diagnostic medical device to assess gastric motility \#: C32-16-00364/ imec funds 2017. C. V. is a postdoctoral fellow of the Research FoundationFlanders (FWO). This paper reflects only the author's views and the Union is not liable for any use that may be made of the contained information.

\section{REFERENCES}

[1] Billman, George E. "Heart rate variabilitya historical perspective." Frontiers in physiology 2 (2011): 86.

[2] Ben-Tal, Alona, et al. "Central regulation of heart rate and the appearance of respiratory sinus arrhythmia: New insights from mathematical modeling." Mathematical biosciences 255 (2014): 71-82.

[3] Morales Tellez, J., et al. "Respiratory sinus arrhythimia in apnea patients with apnea associated comorbidities." Proc. of the 45th Annual Computing in Cardiology (2018): 1-4.

[4] Hellman, JOEL B., and RALPH W. Stacy. "Variation of respiratory sinus arrhythmia with age." Journal of Applied Physiology 41.5 (1976): 734-738.

[5] Faes, Luca, et al. "Investigating cardiac and respiratory determinants of heart rate variability in an information-theoretic framework." 2014 36th Annual International Conference of the IEEE Engineering in Medicine and Biology Society. IEEE, 2014.

[6] Schumann, Aicko Y., et al. "Bivariate phase-rectified signal averaging." Physica A: Statistical Mechanics and its Applications 387.21 (2008): 5091-5100.

[7] Orini, Michele, et al. "A Time-Varying Nonparametric Methodology for Assessing Changes in QT Variability Unrelated to Heart Rate Variability." IEEE Transactions on Biomedical Engineering 65.7 (2018): 1443-1451.

[8] Varon, Carolina, et al. "Unconstrained Estimation of HRV Indices after Removing Respiratory Influences from Heart Rate.” IEEE journal of biomedical and health informatics (2018).

[9] Force, Task. "Standards of measurement, physiological interpretation and clinical use. Task force of the European Society of Cardiology and the North American Society of Pacing and Electrophysiology." Circulation 93.5 (1996): 1043-1065.

[10] Berry, Richard B., et al. "Rules for scoring respiratory events in sleep: update of the 2007 AASM manual for the scoring of sleep and associated events." Journal of clinical sleep medicine 8.05 (2012): 597-619.

[11] Varon, Carolina, et al. "A novel algorithm for the automatic detection of sleep apnea from single-lead ECG." IEEE Transactions on Biomedical Engineering 62.9 (2015): 2269-2278.

[12] Stein, Phyllis K., and Yachuan Pu. "Heart rate variability, sleep and sleep disorders." Sleep medicine reviews 16.1 (2012): 47-66.

[13] Varon, Carolina. "Mining the ECG: Algorithms and Applications." (2015).

[14] Joshi, Rohan, et al. "Cardiorespiratory coupling in preterm infants." Journal of Applied Physiology 126.1 (2018): 202-213.

[15] Angelone, Alfonso, and Norman A. Coulter JR. "Respiratory sinus arrhythmia: a frequency dependent phenomenon.” Journal of Applied Physiology 19.3 (1964): 479-482.

[16] Denver, John W., Shawn F. Reed, and Stephen W. Porges. "Methodological issues in the quantification of respiratory sinus arrhythmia." Biological psychology 74.2 (2007): 286-294. 